\title{
AN IMBEDDING THEOREM FOR ANISOTROPIC ORLICZ-SOBOLEV SPACES
}

\author{
G. HARDY AND H.B. Thompson
}

Let $G$ be a convex function of $m$ variables, let $\Omega$ be a domain in $\mathbb{R}^{n}$, and let $L_{G}(\Omega)$ denote the vector-valued Orlicz space determined by $G$. We give an imbedding theorem for the space $W_{G}^{1}(\Omega)$ of weakly differentiable functions $u$ provided with the norm $\|(u, D u)\|_{G}$, where $m=n+1$ and $D u$ denotes the gradient of $u$. This theorem is a variant of an imbedding theorem by N.S. Trudinger for the completion of $C_{0}^{1}(\Omega)$ in the norm $\|D u\|_{G}$, where $\mathrm{m}=\mathrm{n}$.

\section{Preliminaries}

We shall use the definitions and properties of vector-valued Orlicz spaces as given in [2], with an additional monotonicity requirement. References on Orlicz spaces can be found in [2].

Definition: A function $G: \mathbb{R}^{m} \rightarrow[0, \infty]$ is said to be a $G$-function of $m$ variables if

(i) $G(0)=0$;

(ii) $\lim _{|x| \rightarrow \infty} G(x)=\infty$;

(iii) $G$ is convex;

(iv) $G$ is symmetric;

(v) $G^{-1}(\infty)$ is bounded away from zero;

(vi) $G$ is lower semicontinuous.

$G$ is called a Young function if $m=1$.

We further assume that

(vii) $G$ is monotone increasing in each variable separately.

If $\Omega$ is a domain in $\mathbb{R}^{n}, u_{1}, \ldots, u_{m}$ are measurable on $\Omega$, and $u=\left(u_{1}, \ldots, u_{m}\right)$, then $L_{G}(\Omega)$ is defined by

$$
L_{G}(\Omega)=\left\{u: \int_{\Omega} G(\alpha u) d x<\infty \text { for some } \alpha>0\right\}
$$

Received 25th July, 1994

Copyright Clearance Centre, Inc. Serial-fee code: 0004-9729/95 \$A2.00+0.00. 
Definition: The Luxemburg norm $\|u\|_{G, \Omega}=\|u\|_{G}$ is defined by

$$
\|u\|_{G, \Omega}=\inf \left\{k>0: \int_{\Omega} G(u / k) d x \leqslant 1\right\} .
$$

Definition: The conjugate function $G_{+}^{*}$ of $G$ is defined by

$$
G_{+}^{*}(x)=\sup _{y_{i} \geqslant 0}\{x \cdot y-G(y)\}
$$

For $u \in L_{G}(\Omega), v \in L_{G_{+}^{*}}(\Omega)$, the following generalised Hölder inequality holds:

$$
\int_{\Omega} u \cdot v d x \leqslant 2\|u\|_{G}\|v\|_{G_{+}^{*}} .
$$

DEFINITION: If $G$ is a $G$-function of $n+1$ variables, then $W_{G}^{1}(\Omega)$ denotes the set of weakly differentiable functions $u$ for which $\left(u, D_{1} u, \ldots, D_{n} u\right)=(u, D u)$ belongs to $L_{G}(\Omega)$. A norm is defined on $W_{G}^{1}(\Omega)$ by

$$
\|u\|_{W_{G}^{1}(\Omega)}=\|u\|_{W_{G}^{1}}=\|(u, D u)\|_{G}
$$

Definition: A domain $\Omega \subset \mathbb{R}^{n}$ is said to be admissible if there exists a constant $\gamma$, depending only on $n$, such that, for any $u$ in the Sobolev space $W^{1} L_{1}(\Omega)$,

$$
\|u\|_{n /(n-1)} \leqslant \gamma\left(\|u\|_{1}+\sum_{i=1}^{n}\left\|D_{i} u\right\|_{1}\right) .
$$

(See [1].) It is known that (4) is true if $\Omega$ satisfies the cone condition, that is, if there exists a fixed cone $k_{\Omega} \subset \mathbb{R}^{n}$ such that each point $x$ of $\Omega$ is the vertex of a cone $k_{\Omega}(x) \subset \Omega$, congruent to $k_{\Omega}$.

We shall use the following extension of the chain rule (see [1]):

Lemma. If $u$ is a weakly differentiable function on a domain $\Omega \subset \mathbb{R}^{n}$ and if $g$ is a uniformly Lipschitz continuous function on $\mathbb{R}$, then $g \circ u$ is weakly differentiable, and

$$
D g(u)=g^{\prime}(u) D u \quad \text { almost everywhere in } \Omega,
$$

that is,

$$
D_{i} g(u)=g^{\prime}(u) D_{i} u, \quad i=1, \ldots, n
$$

where, in (5), the convention is made that the right side is zero if $D_{i} u$ is zero, even if $g^{\prime}(u)$ is undefined or infinite. 


\section{AN IMBEDDING THEOREM}

THEOREM. Let $\Omega$ be a bounded admissible domain in $\mathbb{R}^{n}$. Let $G$ be a $G$-function of $n+1$ variables, and suppose $F$ is a continuous, non-negative function on $[0, \infty)$ such that

$$
G_{+}^{*}[0, F(s), \ldots, F(s)] \leqslant s .
$$

Let $m(s)=s^{1 / n} F(s)$, and suppose $A$ is a Young function such that

$$
\int_{0}^{|t|} \frac{d s}{m(s)}=k A^{-1}(|t|)
$$

for some constant $k$. Then there exists a constant $C$, depending only on $n$, such that

$$
\|u\|_{A} \leqslant C\|u\|_{W_{G}^{1}}
$$

for any $u \in W_{G}^{1}(\Omega)$.

Proof: We suppose first that $u \in W_{G}^{1}(\Omega)$ is bounded. Let $\lambda=\|u\|_{A}$. Since $\Omega$ is bounded,

$$
\int_{\Omega} A(u / \lambda) d x=1
$$

From (7) and the definition of $m$,

$$
\left(A^{1-1 / n}\right)^{\prime}=k(1-1 / n) F(A) .
$$

Let $C=A^{1-1 / n}$ and let $g=C(u / \lambda)$. By (5), $g \in W_{L_{1}}^{1}(\Omega)$, and since we assumed $\Omega$ is admissible,

$$
\begin{aligned}
\|g\|_{n /(n-1)} & \leqslant \gamma\left[\int_{\Omega}\left(\sum_{i=1}^{n}\left|D_{i} g\right|\right) d x+\|g\|_{1}\right] \\
& =\frac{\gamma}{\lambda} \int_{\Omega}\left(\sum_{i=1}^{n}\left|C^{\prime}(u / \lambda) D_{i} u\right|\right) d x+\gamma\|g\|_{1} \\
& \leqslant \frac{2 \gamma}{\lambda}\left\|\left(0, C^{\prime}(u / \lambda), \ldots, C^{\prime}(u / \lambda)\right)\right\|_{G_{+}^{*}}\|(u, D u)\|_{G}+\gamma\|g\|_{1}
\end{aligned}
$$

from Hölder's inequality (2). Then using the definition of $C$ and (10), we get (11)

$$
\|g\|_{n /(n-1)} \leqslant \frac{2 \gamma}{\lambda} k(1-1 / n)\|(0, F[A(u / \lambda)], \ldots, F[A(u / \lambda)])\|_{G_{+}^{*}}\|(u, D u)\|_{G}+\gamma\|g\|_{1}
$$


Using (6) and (9), we have

$$
\int_{\Omega} G_{+}^{*}\{(0, F[A(u / \lambda)], \ldots, F[A(u / \lambda)])\} d x \leqslant \int_{\Omega} A(u / \lambda) d x=1
$$

and so

$$
\|(0, F[A(u / \lambda)], \ldots, F[A(u / \lambda)])\|_{G_{+}^{*}} \leqslant 1
$$

from the definition of the Luxemburg norm (1). From the definition of $G$ and from (9),

$$
\|g\|_{n /(n-1)}=\left[\int_{\Omega} A(u / \lambda) d x\right]^{(n-1) / n}=1 .
$$

Since $\frac{g(t / \lambda) /(t / \lambda)}{A(t / \lambda) /(t / \lambda)}=\frac{1}{A^{1 / n}(t / \lambda)} \rightarrow 0$ as $t \rightarrow \infty$, there exists $t_{0}$ such that

$$
\frac{g(t / \lambda)}{t / \lambda} \leqslant \frac{1}{2 \gamma} \frac{A(t / \lambda)}{t / \lambda}
$$

for $t \geqslant t_{0}$. Further, by L'Hospital's rule, $g(t / \lambda) \rightarrow 0$ as $t \rightarrow 0$, so that $K=$ $\sup [g(t / \lambda) /(t / \lambda)]$ is finite. Thus, for all $t>0$, $t \in\left(0, t_{0}\right)$

$$
g(t / \lambda) \leqslant \frac{1}{2 \gamma} A(t / \lambda)+K t / \lambda
$$

Replacing $t$ in the last equation by $u$, integrating over $\Omega$, and using (9) gives

$$
\|g\|_{1} \leqslant 1 /(2 \gamma)+(K / \lambda)\|u\|_{1} .
$$

Thus from (11), (12), (13), and (14) we obtain

$$
1 \leqslant \frac{2 \gamma}{\lambda}\|(u, D u)\|_{G}+\frac{1}{2}+\frac{\gamma K}{\lambda}\|u\|_{1},
$$

that is,

$$
\lambda \leqslant 4 \gamma\|(u, D u)\|_{G}+2 \gamma K\|u\|_{1}
$$

But

$$
\|u\|_{1} \leqslant 2\|(1,0, \ldots, 0)\|_{G_{+}^{*}}\|(u, D u)\|_{G} ;
$$

hence (15) may be written as

$$
\|u\|_{A} \leqslant C\|(u, D u)\|_{G},
$$

where $C$ depends only on $n$.

If $u$ is not bounded, we define

$$
u_{l}= \begin{cases}u, & \text { for }|u|<l \\ l \operatorname{sign} u, & \text { for }|u| \geqslant l .\end{cases}
$$

By the chain rule (5), $u_{l}$ belongs to $W_{G}^{1}(\Omega)$, and by the monotone convergence theorem, $\left\|u_{l}\right\|_{A} \rightarrow\|u\|_{A}$ and $\left\|\left(u_{l}, D u_{l}\right)\right\|_{G} \rightarrow\|(u, D u)\|_{G}$, so that (16) is true for all $u \in W_{G}^{1}(\Omega)$. Referring to (3), we see that this establishes (8), so the theorem is proved. 


\section{References}

[1] T.K. Donaldson and N.S. Trudinger, 'Orlicz-Sobolev spaces and imbedding theorems', $J$. Funct. Anal. 8 (1971), 52-75.

[2] N.S. Trudinger, 'An imbedding theorem for $H^{\circ}(G, \Omega)$ spaces', Studia Math. 50 (1974), 17-30.

Department of Mathematics

The University of Queensland

Queensland 4072

Australia 